\title{
La Correspondance de Condorcet. Documents inédits, nouveaux éclairages. Engagements politiques 1775-1792, textes réunis et présentés par Nicolas Rieucau
}

\section{Marisa Ferrarini}

\section{(2) OpenEdition \\ 1 Journals}

\section{Edizione digitale}

URL: http://journals.openedition.org/studifrancesi/1178

DOI: 10.4000/studifrancesi. 1178

ISSN: 2421-5856

\section{Editore}

Rosenberg \& Sellier

\section{Edizione cartacea}

Data di pubblicazione: 1 novembre 2014

Paginazione: 600-601

ISSN: 0039-2944

\section{Notizia bibliografica digitale}

Marisa Ferrarini, «La Correspondance de Condorcet. Documents inédits, nouveaux éclairages.

Engagements politiques 1775-1792, textes réunis et présentés par Nicolas Rieucau », Studi Francesi

[Online], 174 (LVIII | III) | 2014, online dal 01 novembre 2014, consultato il 18 septembre 2020. URL

http://journals.openedition.org/studifrancesi/1178; DOI : https://doi.org/10.4000/studifrancesi. 1178

Questo documento è stato generato automaticamente il 18 settembre 2020.

\section{cc) (†) $\odot$}

Studi Francesi è distribuita con Licenza Creative Commons Attribuzione - Non commerciale - Non opere derivate 4.0 Internazionale. 


\title{
La Correspondance de Condorcet. Documents inédits, nouveaux éclairages. Engagements politiques 1775-1792, textes réunis et présentés par Nicolas Rieucau
}

\author{
Marisa Ferrarini
}

\section{NOTIZIA}

La Correspondance de Condorcet. Documents inédits, nouveaux éclairages. Engagements politiques 1775-1792, textes réunis et présentés par Nicolas RIEUCAU, avec la collaboration d'Annie CHASSAGNE et Christian GILAIN, analyse matérielle: Claire BUSTARRET, FerneyVoltaire, Centre international d'étude du XviII ${ }^{\mathrm{e}}$ siècle, 2014, pp. 240.

1 Il volume in questione riunisce la maggior parte degli interventi presentati alla giornata di studio La correspondance de Condorcet: documents inédits, nouveaux éclairages, tenutasi a Parigi, presso il Centre Panthéon-Sorbonne, l'11 maggio 2012, e s'inscrive nel quadro più generale del progetto Inventaire analytique et matériel de la correspondance de Condorcet, a cui da tempo sta lavorando un'équipe internazionale e pluridisciplinare (l'elenco dei partecipanti è consultabile all'indirizzo www.inventaire-condorcet.com/ fra/page/7). All'inizio dell'opera sono illustrati i problemi specifici del progetto (dispersione dei documenti, identificazione dei corrispondenti, datazione delle lettere) e i metodi utilizzati per risolverli, tra i quali l'analisi materiale: studio della carta, dello spazio grafico, dell'inchiostro, della forma della scrittura, della grafia, delle iscrizioni postali e dei sigilli di ceralacca.

2 Come indica il sottotitolo, Engagements politiques 1775-1792, la corrispondenza di Condorcet commentata, che si basa su più di centocinquanta documenti inediti, tra cui 
una trentina di lettere pubblicate per la prima volta, è sistematicamente connessa alle battaglie politiche del proprio tempo e nello specifico la navigazione fluviale, l'edizione Kehl delle opere complete di Voltaire, l'uniformazione dei pesi e delle misure, la Costituzione francese e americana.

3 La partecipazione al ministero Turgot (1774-1776) costituisce per l'illustre accademico una prima esperienza concreta dei rapporti tra teoria e pratica, soprattutto in materia di lavori pubblici, moneta, fiscalità. Incentrato sullo sviluppo dei canali, il contributo di Éric Szulman (Aux sources d'une activité administrative: la correspondance entre Condorcet et Trudaine au sujet de la navigation intérieure sous le ministère Turgot, pp. 39-62) mostra come il giovane Condorcet si batta con ardore per modernizzare la rete amministrativa, tecnica, economica e finanziaria collegata alla circolazione fluviale. A suo dire, questa dovrebbe essere orchestrata da un governo di saggi che dettino ordini e raccomandazioni agli ingegneri dei Ponts et chaussés. Il suo tentativo purtroppo non andrà a buon fine, ma l'esperienza amministrativa sfocerà nella redazione del Mémoire sur le canal de Picardie (1780).

4 Alla luce di tre lettere inedite della corrispondenza di Condorcet con Brissot, Calogero Alberto PETIX evidenzia la natura pragmatica e di conseguenza evolutiva della sua riflessione sulla costituzione (Vers le républicanisme: l'évolution de la pensée politique de Condorcet à la lumière de sa correspondance inédite avec Brissot, pp. 63-81). Subito dopo la presa della Bastiglia, l'enciclopedista teme che la Francia sprofondi nel caos politico, infatti il 7 agosto 1789 scrive a Brissot che «il est aussi necessaire pour le moins dans ce moment ci d'éclairer le peuple sur les limites de ses droits que sur leur étendue» (p. 74). Ristabilire l'ordine pubblico e insegnare il rispetto della legge sono, in quest'ottica, condizioni preliminari per estendere il diritto di cittadinanza. All'inizio del 1790, Condorcet accetta che la nazione possa rimettere in discussione il potere monarchico, ma solo dopo l'episodio di Varennes auspica l'instaurazione della repubblica. Questo prova che le sue riflessioni sono spesso dettate dall'esigenza di trovare una soluzione pratica a un problema immediato. Le lettere analizzate hanno inoltre il merito d'illuminarci sulla natura del rapporto tra Condorcet e Brissot, sinora basato su documenti di seconda mano.

5 Manuela ALBERTONE analizza la corrispondenza di Condorcet con alcuni interlocutori americani, in particolare Benjamin Franklin e Thomas Jefferson (Condorcet et ses correspondants américains: de grands interlocuteurs et de petites découvertes, pp. 83-107). Le lettere a Franklin, nel periodo 1773-1778, sono contrassegnate da contatti cordiali, ma non rivelano una vera familiarità: Franklin è solo un intermediario per accedere agli ambienti americani. Maggiore è l'affinità con Jefferson. Lo scambio tra i due appare inoltre più nettamente politico, persino nelle lettere concernenti l'uniformazione dei pesi e delle misure.

6 Gli ultimi due contributi illustrano, attraverso documenti inediti, il ruolo di direttore scientifico assunto da Condorcet nella preparazione dell'edizione Kehl delle opere complete di Voltaire. Come spiega Linda GIL (Condorcet, éditeur de Voltaire: une correspondance en réseau, pp. 111-137), questo lavoro è soprattutto un lavoro d'équipe, che si costituisce rapidamente in una rete di corrispondenti di dimensione locale, nazionale ed europea, in cui Condorcet occupa il posto centrale. Il saggio di André MAGNAN si concentra invece sullo scambio tra Condorcet e Ruault, segretario e intermediario tra i diversi collaboratori del progetto (La navette Condorcet-Ruault archive central du «Voltaire» de Kehl, pp. 139-202). 
7 Il volume si chiude in bellezza con le testimonianze inedite di Ruault su Condorcet, scoperte nelle sue lettere a Jacques-Joseph Decroix (André MAGNAN, condorcet vu par Ruault, pp. 203-225). Evidenti sono l'affetto e la stima per colui che dopo Voltaire, dopo d'Alembert, deve essere e sarà il "général», il «chef de la philosophie», «notre capitaine» o il levita che scrive la vita del Mosé dei tempi futuri (p.203).

8 Come si può facilmente intuire, questo volume è uno strumento prezioso che permette di apprezzare in modo più preciso le idee e l'azione di Condorcet, rivelando l'ampiezza e la varietà dei suoi rapporti con i contemporanei (scienziati, filosofi, politici, amministratori), e di approfondire la nostra conoscenza sull'epoca dei Lumi e della Rivoluzione francese. 